\title{
Role of honeycomb structure in improving the melting process of a phase change material inside a latent heat storage unit
}

\author{
M. Hariss ${ }^{1}$, M. El Alami ${ }^{1}$ and A. Gounni ${ }^{1}$ \\ ${ }^{1}$ Physics Department \\ LPMMAT Laboratory \\ Faculty of Sciences Ain Chock, Hassan II University \\ Casablanca, Morocco \\ Phone: +212 617703386, e-mail: harissmanal@gmail.com, elalaminus@gmail.com,gounni.ayoub@gmail.com
}

\begin{abstract}
In this work, a numerical study is performed to analyze the impact of honeycomb structure on heat transfer within the PCM. The modeling is based on a transient calculation making it possible to analyze the phase change of the paraffin using the commercial software "Fluent" based on the enthalpy-porosity model. The results showed that the impregnation of a metal matrix in a rectangular enclosure helps to decrease the melting time and thus improve the heat transfer within the PCM.
\end{abstract}

Keywords. Phase change material, latent heat thermal energy storage, CFD, honeycomb, Fusion.

\section{Introduction}

In the last decades, the large increase and the deterioration of the climate conditions and fossil fuels have promoted several studies on developing a new strategy to curb the ongoing situation. As a result, clean and renewable energy has become an unwavering alternative to alleviate the current dilemma. Nevertheless, the intermittent behavior of clean energy hampers its applicability. Thermal energy storage (THS) technology presents an effective way to mitigate the sporadic nature of renewable energy. Between the major types of TES, latent heat thermal energy storage of (LHTES) has the advantage of storing the heat due to high energy storage and small temperature variation, the benefits are attributed to the usage of PCM. Despite that, the phase change materials suffer from a low thermal conductivity which hinders the rate of charging and discharging.

To tackle this, different studies are examining different approaches, including the extension of the heat transfer zone using fins, metal matrix, carbon nanotube, and the use of nanoparticles [1],[2].

X. Meng et al.[3] adopted a two-dimensional numerical model to analyze the influence of the porosity as well as the pore density of copper foam on the thermal behavior of the composite (copper foam+ paraffin). The results indicate that the thermal behavior of PCM is improved as the porosity is reduced or as the pore density is increased. To understand the role of pin fin- metal foam composite structure on the solidification of a phase change material, a numerical and experimental study was developed by $\mathrm{X}$. Yang et al.[4]. For this, three-dimensional numerical simulations were carried out and verified with experimental measurements. The results obtained showed that the fin-foam composite promotes the improvement of the solidification compared to competing structures (pin fin \& metal foam).

Qureshi et al.[5] have proposed finned metal foam-PCM systems based on triply periodic minimum surface structures (Primitive, IWP, Gyroid) to study and compare their performance with conventional metallic foam (Kelvin cell) in pure conduction and natural convection. According to the results, metal foams based on TPMS are very attractive for application in TES systems because they exhibited improved heat transfer performance.

An experimental study was developed by M.A. Hayat et al.[6] to inspect the thermal behavior of different heat sinks with PCM (RT-35HC) based on the application of copper foam and heat pipes by imposing three values of heat flux $\left(2.2 .5,3 \mathrm{KW} / \mathrm{m}^{2}\right)$. The results revealed that hybrid cooling $(\mathrm{CF}+\mathrm{HP}+\mathrm{PCM})$ can reduce the base temperature of the heat sink by $47 \%, 51 \%$, and $54 \%$ for

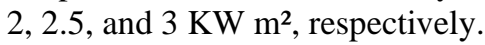

To remedy this problem, we propose a numerical study based on the numerical simulation of the fusion of a phase change material within a rectangular enclosure with a honeycomb matrix.

\section{Numerical procedure}

\section{A. Assumptions}

Transient two-dimensional model of heat transfer during PCM melting process was governed by the following general assumptions:

- Thermophysical properties of PCM are constant for the temperature. 
- The fluid is Newtonian and incompressible;

- We neglect convection in the liquid phase due to the great value of the PCM viscosity.

\section{B. Heat Equation}

The only mode of heat transfer considered during the fusion process is conduction. The effects of natural convection at the solid-liquid interface are neglected. The guiding equation considered here is the classical heat equation:

$$
\rho C_{p} \frac{\partial T}{\partial t}+\operatorname{div}(-k \overrightarrow{\nabla T})=0
$$

\section{Discretization Method}

A two-dimensional transient model based on the finite volume method using an enthalpy-porosity technique is implemented to analyze the performance of a thermal energy storage unit within a rectangular metal matrix enclosure.

The CFD method was used to solve and calculate the governing equations. The Second Order Upwind scheme and the SIMPLE method for pressure-velocity coupling are used to solve the momentum and energy equations. The "Second-order" scheme is also adopted for the pressure correction equation.

\section{Validation}

To ensure the accuracy of the current numerical approach, the average liquid fraction is verified with the experimental results of H.Shokouhmand, B. Kamkari[7]. The lauric acid is filled into a rectangular enclosure with an inside dimension of $120 \mathrm{~mm}$ in height and $50 \mathrm{~mm}$ in width \& depth. The PCM is initially at a constant temperature of $299 \mathrm{~K}$. The right wall of the cavity is subjected to a constant temperature $\left(70^{\circ} \mathrm{C}\right)$ while adiabatic boundary conditions are set on the other walls. Figure 1 depicts a comparison of numerical results (present study) and experimental results of melt fraction variation for a constant temperature. It can be seen that the numerical model is very consistent with the experiment results.

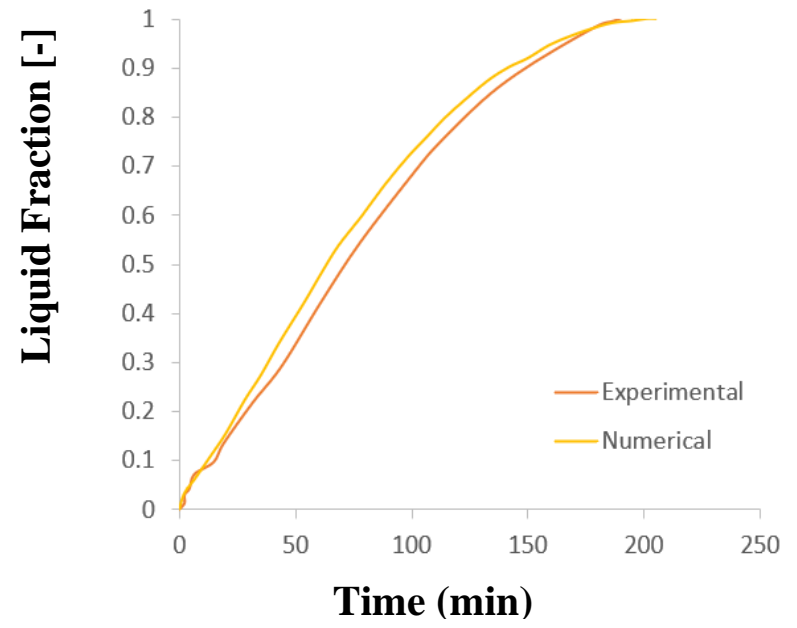

Fig.1. Comparison of numerical results (present study) and experimental results (H.Shokouhmand, B.

Kamkari[7])

\section{Results and Discussions}

\section{A. Studied configuration}

The main objective of this study was to numerically study the heat transfer during the PCM fusion process in an enclosure with and without the honeycomb. This study was motivated by the need to better understand the heat transfer during the charging phase of the thermal energy storage system, which takes advantage of the latent heat of fusion of PCM.

The initial temperature of PCM is set to be slightly lower than the melting temperature $(298 \mathrm{~K})$, so the liquid phase fraction $\lambda=0$. For the two-dimensional model (Figure 2), the bottom wall is subjected to constant heat flux, the left and right sides (indicated in red) are set as adiabatic, while the prescribed convection conditions are imposed on the top horizontal wall.

In addition, we propose in parallel a comparison between two configurations (represented in Figure 2) subjected to the same boundary conditions.

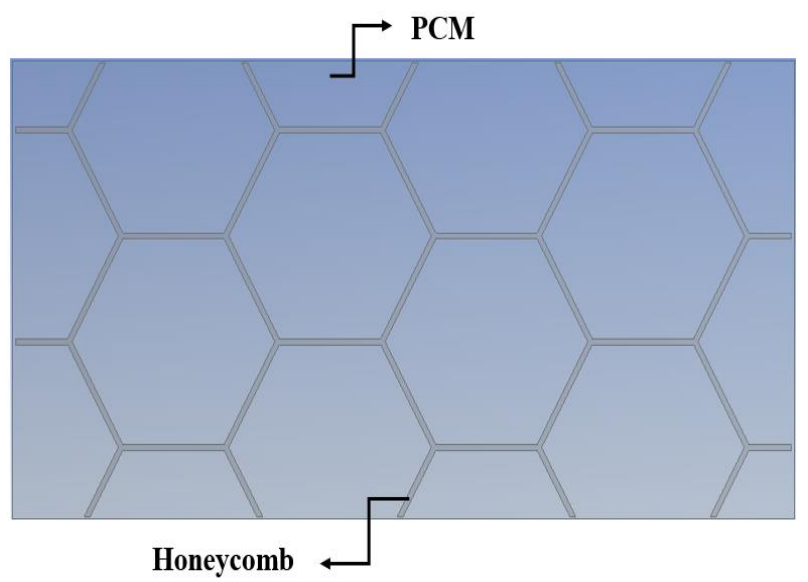

(a)

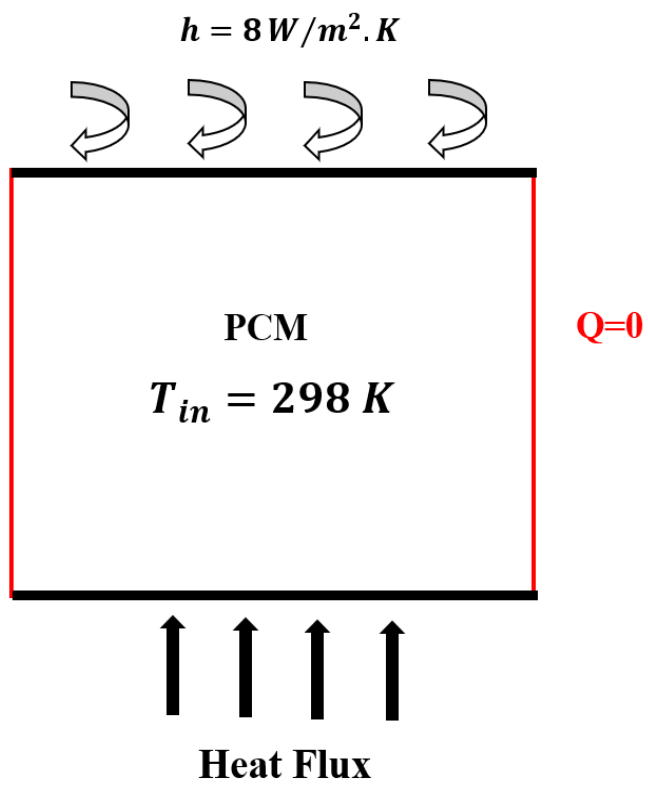

(b)

Fig.2. Rectangular enclosure $(a)$ with honeycomb $(b)$ without honeycomb. 
In this study, an aluminum honeycomb structure was adopted; Table I. represents the thermo-physical properties of aluminum used in the numerical model.

Table I. - Thermophysical properties of aluminum used in the numerical model

\begin{tabular}{|c|c|c|}
\hline PROPERTY & UNITS & VALUE \\
\hline$\rho$ & $\mathrm{kg} / \mathrm{m}^{3}$ & 2719 \\
\hline$\lambda$ & $W / \mathrm{m} . \mathrm{K}$ & 202.2 \\
\hline$C_{p}$ & $\mathrm{l} / \mathrm{kg} \cdot \mathrm{K}$ & 871 \\
\hline
\end{tabular}

The phase change material used in this study is eicosane. Table II. represents the different thermophysical properties used in the numerical simulation.

Table II. - Thermophysical properties of eicosane[8]

\begin{tabular}{|c|c|c|}
\hline PROPERTY & UNITS & VALUE \\
\hline $\boldsymbol{\rho}$ & $\mathbf{k g} / \boldsymbol{m}^{\mathbf{3}}$ & 790 \\
\hline $\boldsymbol{\lambda}$ & $\boldsymbol{W} / \boldsymbol{m} \cdot \boldsymbol{K}$ & 0.23 \\
\hline $\boldsymbol{L}$ & $\boldsymbol{I} / \mathbf{k g}$ & 241000 \\
\hline $\boldsymbol{C}_{\boldsymbol{p}}$ & $\boldsymbol{I} \mathbf{k g} \cdot \boldsymbol{K}$ & 2050 \\
\hline $\boldsymbol{\beta}$ & $\mathbf{1} / \boldsymbol{K}$ & 0.0001 \\
\hline $\boldsymbol{\mu}$ & $\boldsymbol{k g} / \boldsymbol{m} \cdot \boldsymbol{s}$ & 0.00355 \\
\hline $\boldsymbol{T}_{\boldsymbol{m}}$ & $\boldsymbol{K}$ & 309.5 \\
\hline $\boldsymbol{T}_{s}$ & $\boldsymbol{K}$ & 308.5 \\
\hline $\boldsymbol{T}_{\boldsymbol{l}}$ & $\boldsymbol{K}$ & 310.5 \\
\hline
\end{tabular}

\section{B. Phase Change Interface}

Figure 3 shows the variation of the liquid fraction over time. We can easily examine the shape and movement of the fusion interface over time. The red and blue colors represent the condition when the material is completely liquid ( $\mathrm{f}=1$ ) and solid ( $\mathrm{f}=0$ ), respectively. The mushy zone represents the molten front; it separates the liquid and solid regions. We can observe that at the start, $t=0$ $500 \mathrm{~s}$ of the fusion process, the fusion interface starts from bottom to top, near the source (constant heat flux). This indicates that, in the early stages, heat transfer occurs mainly by conduction. We also note that the evolution of fusion begins with the edges of the core of a honeycomb and propagates towards the middle of the hexagon. After 3000s, all of the PCM was melted; giving a melting fraction of close to $100 \%$ for the first configuration (with metal matrix), however, the fraction was still not completed in the second case.

\section{Evolution of the liquid fraction}

The liquid fraction is a typical dimensionless parameter that characterizes the charging characteristics of LHS units. Figure.4. depicts the variation of liquid fraction for eicosane in a rectangular enclosure with and without honeycomb structure during the melting process. In the early stage, we note that the evolution of liquid fraction is similar and linear for both configurations and the heat transfer is mainly by conduction, this is proven in figure 3.

Without honeycomb
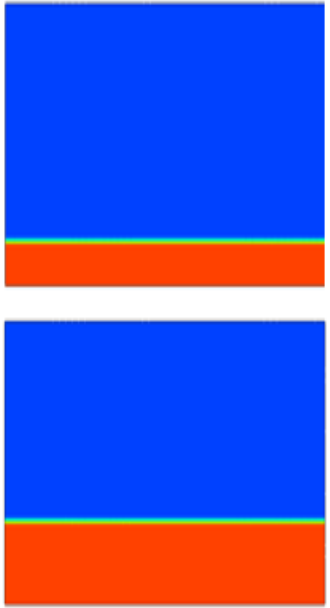

$1000 \mathrm{~s}$

$500 s$

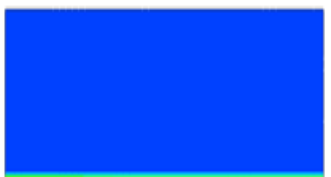

$1500 s$
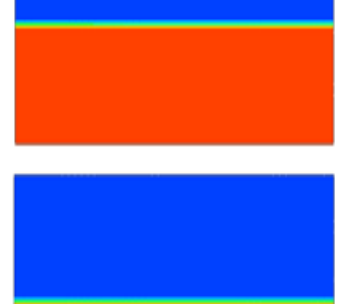

$2000 s$
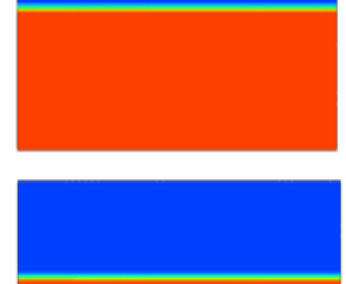

$2500 s$
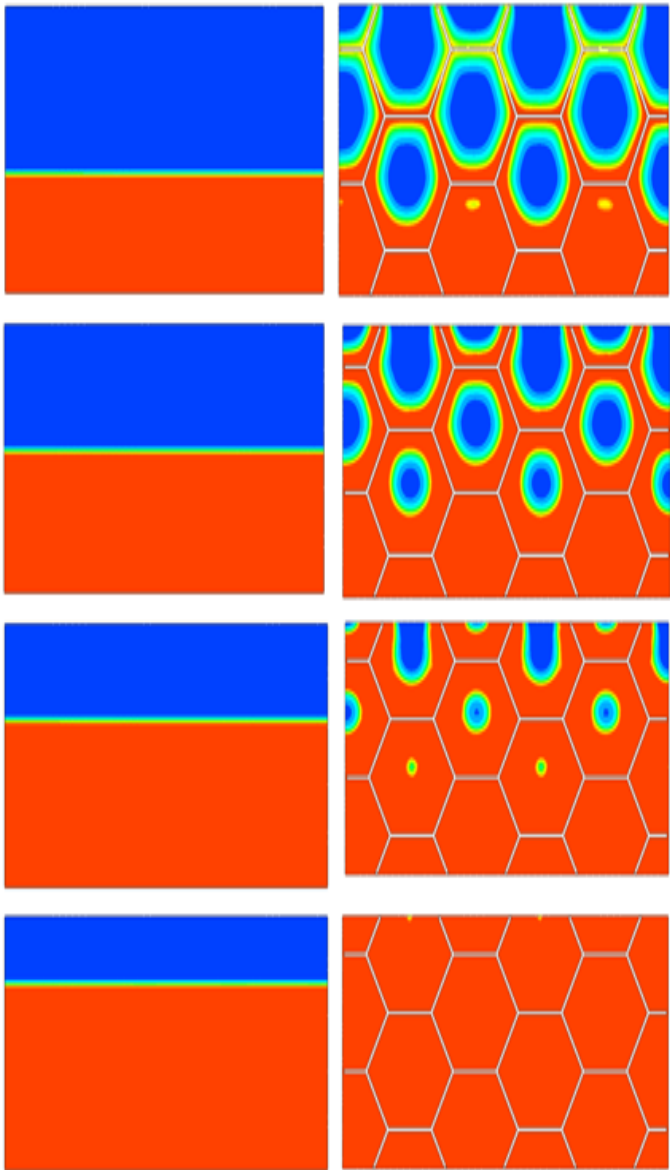

With honeycomb
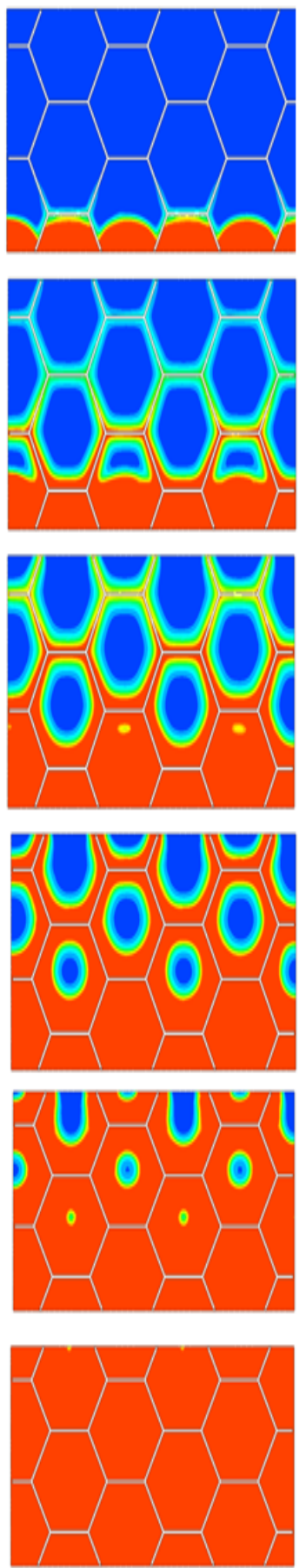

Fig. 3. Graphic representation of the spatial distribution of the liquid fraction $(=1$, red, in the fluid; $=0$, blue, in the solid). 
As shown in figure.4, the increase in the rate of liquid fraction for the LHS unit with the metallic matrix is faster than the unit with the pure PCM. Furthermore, the impregnation of the honeycomb structure makes it possible to reduce the complete melting time by $31 \%$.

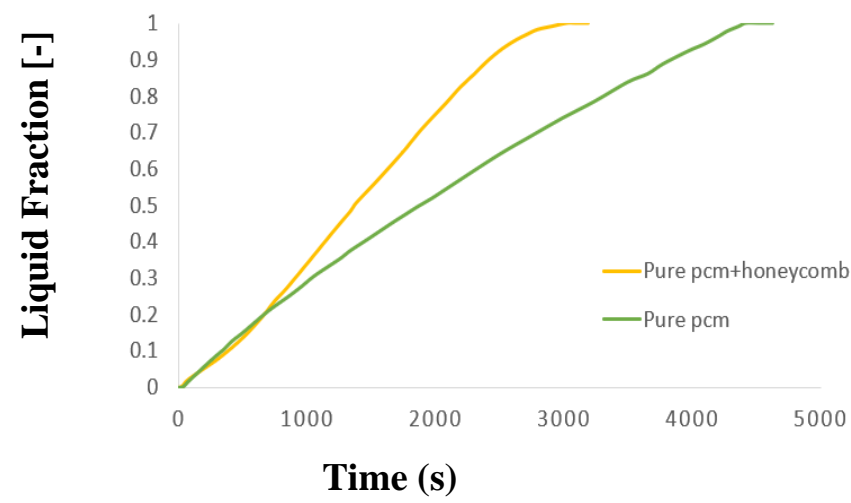

Fig.4. Evolution of liquid fraction

\section{Conclusion}

The work presented aims to understand the phenomenology of thermal energy transfers within a rectangular enclosure with and without conductive honeycomb structures. The numerical results developed by the Fluent code for the two configurations studied, show on the one hand that the phenomenology of the phase change is the same. Only the calculated characteristic times will distinguish one test, faster, from another, slower. On the other hand, we see that the addition of a metal matrix allows to increasing and improving the performance of the system, more precisely the charging time which is a major factor in the field of thermal storage.

\section{References}

[1] L. Liu, D. Su, Y. Tang, et G. Fang, « Thermal conductivity enhancement of phase change materials for thermal energy storage: A review », Renew. Sustain. Energy Rev., vol. 62, p. 305-317, sept. 2016, doi: 10.1016/j.rser.2016.04.057.

[2] Z. A. Qureshi, H. M. Ali, et S. Khushnood, « Recent advances on thermal conductivity enhancement of phase change materials for energy storage system: A review », Int. J. Heat Mass Transf., vol. 127, p. 838-856, déc. 2018, doi: 10.1016/j.ijheatmasstransfer.2018.08.049.

[3] X. Meng, L. Yan, J. Xu, F. He, H. Yu, et M. Zhang, «Effect of porosity and pore density of copper foam on thermal performance of the paraffin-copper foam composite PhaseChange Material », Case Stud. Therm. Eng., vol. 22, p. 100742, déc. 2020, doi: 10.1016/j.csite.2020.100742.

[4] X. Yang, Z. Niu, J. Guo, Q. Bai, H. Li, et Y.-L. He, «Role of pin fin-metal foam composite structure in improving solidification: Performance evaluation », Int. Commun. Heat Mass Transf., vol. 117, p. 104775, oct. 2020, doi: 10.1016/j.icheatmasstransfer.2020.104775.

[5] Z. A. Qureshi, E. Elnajjar, O. Al-Ketan, R. A. Al-Rub, et S. B. Al-Omari, « Heat transfer performance of a finned metal foam-phase change material (FMF-PCM) system incorporating triply periodic minimal surfaces (TPMS) », Int. J. Heat Mass
Transf., vol. 170, p. 121001, mai 2021, doi: 10.1016/j.ijheatmasstransfer.2021.121001.

[6] M. A. Hayat, H. M. Ali, M. M. Janjua, W. Pao, C. Li, et M. Alizadeh, «Phase change material/heat pipe and Copper foam-based heat sinks for thermal management of electronic systems », J. Energy Storage, vol. 32, p. 101971, déc. 2020, doi: 10.1016/j.est.2020.101971.

[7] H. Shokouhmand et B. Kamkari, «Experimental investigation on melting heat transfer characteristics of lauric acid in a rectangular thermal storage unit », Exp. Therm. Fluid Sci., vol. 50, p. 201-212, oct. 2013, doi: 10.1016/j.expthermflusci.2013.06.010.

[8] A. Arshad, M. Jabbal, P. T. Sardari, M. A. Bashir, H. Faraji, et Y. Yan, «Transient simulation of finned heat sinks embedded with PCM for electronics cooling », Therm. Sci. Eng. Prog., vol. 18, p. 100520, août 2020, doi: 10.1016/j.tsep.2020.100520. 